\title{
An exploratory study of the McKay airway to assist bag-mask ventilation
}

\author{
William P. McKay, MD • Udoka Okpalauwaekwe, MD • Cuylar Conly, MSc • \\ Bhavna Pooni, RN, BSN
}

Received: 27 January 2020/Revised: 21 April 2020/Accepted: 24 April 2020/Published online: 8 May 2020

(C) Canadian Anesthesiologists' Society 2020

To the Editor,

We recently described a novel oral airway device, the McKay airway, designed for maintenance of jaw thrust (Figure 1) by first responders performing bag-mask ventilation (BMV). ${ }^{1,2}$ When infrequently performed, BMV is generally not done well., With University of Saskatchewan ethics approval (\#1324; 17 July 2019), we compared the time for naïve learners to achieve target exhaled tidal volume (VE) $>300 \mathrm{~mL}$ during BMV on anesthetized patients using either a Guedel or McKay airway. ${ }^{5}$

The order-randomized cross-over study (performed from 1 August to 18 December 2019) enrolled 31 consenting learners (medical students, non-anesthesia residents, and other naïve learners scheduled in the operating rooms to be taught airway management) who performed BMV on one

Electronic supplementary material The online version of this article (https://doi.org/10.1007/s12630-020-01696-3) contains supplementary material, which is available to authorized users.

W. P. McKay, MD ( $\bowtie)$.

Department of Anesthesia, University of Saskatchewan,

Saskatoon, SK, Canada

e-mail: bill.mckay@usask.ca

U. Okpalauwaekwe, MD

Division of Cardiology, University of Saskatchewan, Saskatoon,

SK, Canada

C. Conly, MSc

University of Saskatchewan College of Medicine, Saskatoon, SK, Canada

B. Pooni, RN, BSN

Faculty, Perioperative Services and Medical Device

Reprocessing, Saskatchewan Polytechnic Institute, Saskatoon,

SK, Canada or more patients. Consenting adults undergoing elective surgery under general anesthesia were recruited. Exclusion criteria included temporomandibular joint disorders, loose incisors, reflux, and a attending anesthesiologist's opinion of any added patient risk for participation. For edentulous patients, we padded the McKay airway surface with a $1-\mathrm{cm}$ thick stick-on sponge (Reston ${ }^{\mathrm{TM}}$ Self-Adhering Foam Pads; 3 M, Maplewood, MN, USA).

Following induction of anesthesia and initial BMV by the attending anesthesiologist, an opaque numbered envelope was opened to randomly assign which airway was to be used first. We simulated a self-inflating BMV device by setting the adjustable pressure limit valve on the anesthetic machine to $>30 \mathrm{~cm}$ water pressure and using the oxygen flush to ensure that the reservoir bag was filled between breath attempts. After the airway was inserted, learners applied the mask with their left hand, squeezing the bag with their right approximately every three seconds. The time was recorded from the first bag squeeze until the primary outcome of a VE $>300 \mathrm{~mL}$ was reached. The first airway was then removed and replaced with the alternate airway and the procedure repeated. The anesthesiologist's intervention (if needed) and comments were recorded. Time measurement differences that were non-normally distributed were analysed with a paired t-test of the logtransformed data that passed Shapiro-Wilk testing for normality; all other normally distributed variables were compared using a paired t-test. Incidences were analysed with McNemar's test or Chi squared test for paired and unpaired data, respectively.

We enrolled 34 female and 20 male patients and obtained data from 51. One obese patient had severe succinylcholine fasciculations with rapid oxygen desaturation and the attending anesthesiologist took over immediately and intubated. For two others, the attending 
Figure 1 A) $X$-ray showing pharyngeal airway anteroposterior width (arrows) without McKay airway. B) McKay airway with teeth in grooves to maintain jaw thrust. C) X-ray showing pharyngeal airway anteroposterior width with McKay airway (red arrow) in place and jaw thrust maintained. Upward movement of the jaw is indicated by molars marked with dashed lines

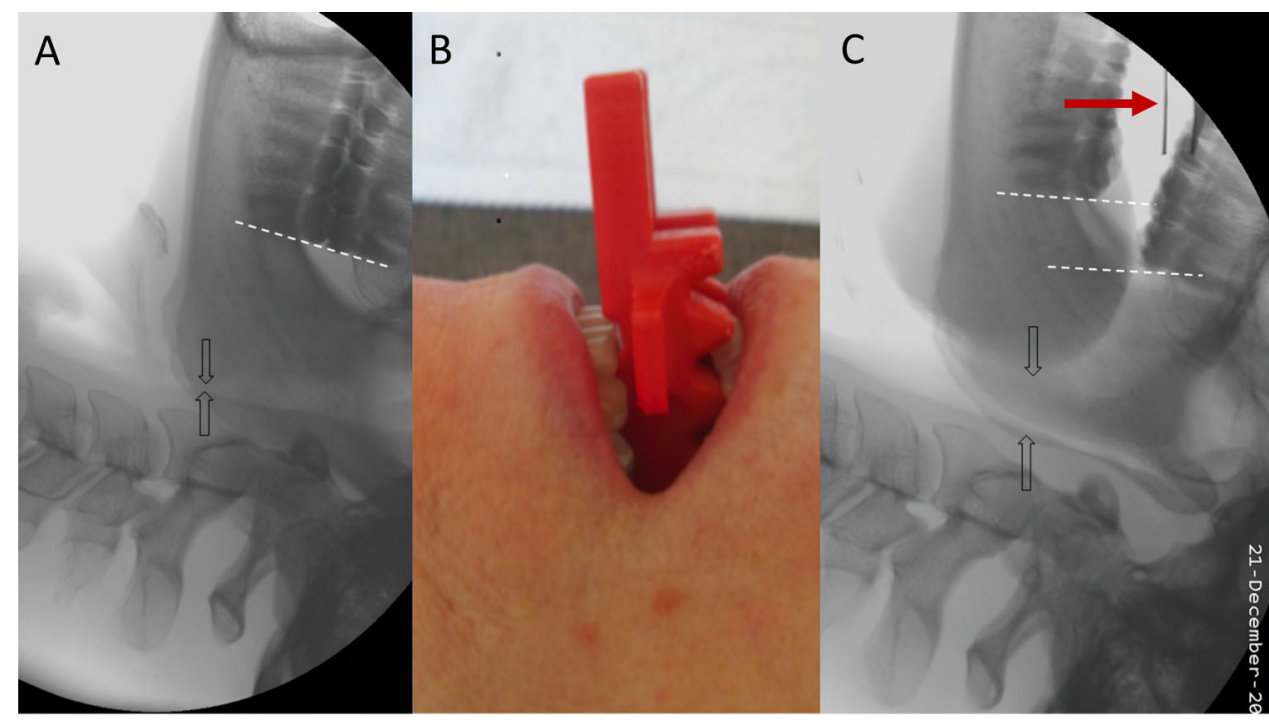

anesthesiologist misunderstood the study protocol. The McKay airway resulted in a shorter mean (standard deviation [SD]) time to achieve target ventilation compared with the Guedel airway [11 (2) vs 14 (2) sec, respectively; mean difference, $3 ; 95 \%$ confidence interval (CI), 2 to $5 ; P=0.011]$. In an exploratory post hoc analysis, the Kaplan-Meier graphs (eAppendix in the Electronic Supplementary Material) show that while the time to VE is very similar in the two thirds of patients that reach target ventilation the soonest, for the third that were presumably more difficult to ventilate, the differences are greater [22 (14.3) sec McKay vs 38 (15.1) sec Guedel; mean difference, $16 ; 95 \% \mathrm{CI}, 7$ to $25 ; P=0.002]$. Comments from participants revealed that 16 preferred the McKay airway, two the Guedel airway $(P<0.001)$, with 15 having no preference.

Participants were randomized to use either the McKay airway first (28 times) or the Guedel airway first (26 times). Overall, attempts with the second device resulted in a shorter mean (SD) time to reach the VE target than with the first [14 (2) vs 11 (2) sec; mean difference, 3; 95\% CI, 2 to $4 ; P=0.03)$. This was probably due to a learning effect as learners became more familiar with BMV technique. Despite this, when used first, the McKay airway was faster (faster/slower: 14/37 times vs 6/45), $P<0.001$ ) than the Guedel airway. Thus, the McKay airway may be easier for beginners to learn. Further studies are suggested.
Disclosures W.P. McKay, U. Okpalauwaekwe, and C. Conly have a vested interest in this study (US patent application 16/098,530 owned by University of Saskatchewan).

Funding statement None.

Editorial responsibility This submission was handled by Dr. Hilary P. Grocott, Editor-in-Chief, Canadian Journal of Anesthesia.

\section{References}

1. McKay WP. Description of a novel oral airway: the McKay airway. Can J Anesth. 2020. DOI: https://doi.org/10.1007/s12630020-01626-3.

2. McKay WP. The McKay Airway for Effective Ventilation. Soteria Medtech. Available from URL: https://soteriamedtech.com/ (accessed April 2020).

3. Soleimanpour H, Gholipouri C, Panahi JR, et al. Role of anesthesiology curriculum in improving bag-mask ventilation and intubation success rates of emergency medicine residents: a prospective descriptive study. BMC Emerg Med. 2011. DOI: https://doi.org/10.1186/1471-227X-11-8.

4. Timmermann A, Russo SG, Crozier TA, et al. Novices ventilate and intubate quicker and safer via intubating laryngeal mask than by conventional bag-mask ventilation and laryngoscopy. Anesthesiology. 2007;107:570-6.

5. Baskett TF. Arthur Guedel and the oropharyngeal airway. Resuscitation. 2004;63:3-5.

Publisher's Note Springer Nature remains neutral with regard to jurisdictional claims in published maps and institutional affiliations. 